JOURNAL of

MAINE MEDICAL CENTER Journal of Maine Medical Center

\title{
Trends in Diagnoses of Neonatal Abstinence Syndrome at Newborn Hospitalization in Maine, 2009-2018
}

\author{
Emily C. Bauer MPH \\ University of Southern Maine
}

Et al.

Follow this and additional works at: https://knowledgeconnection.mainehealth.org/jmmc

Part of the Health Services Research Commons, Maternal and Child Health Commons, and the Women's Health Commons

\section{Recommended Citation}

Bauer, Emily C. MPH; Ahrens, Katherine PhD; and Carwile, Jenny L. MPH, ScD (2021) "Trends in Diagnoses of Neonatal Abstinence Syndrome at Newborn Hospitalization in Maine, 2009-2018," Journal of Maine Medical Center. Vol. 3 : Iss. 1 , Article 4.

Available at: https://knowledgeconnection.mainehealth.org/jmmc/vol3/iss1/4 https://doi.org/10.46804/ 2641-2225.1071

The views and thoughts expressed in this manuscript belong solely to the author[s] and do not reflect the opinions of the Journal of Maine Medical Center or MaineHealth.

This Original Research is brought to you for free and open access by Maine Medical Center Department of Medical Education. It has been accepted for inclusion in the Journal of Maine Medical Center by an authorized editor of the MaineHealth Knowledge Connection. For more information, please contact Dina McKelvy mckeld1@mmc.org.

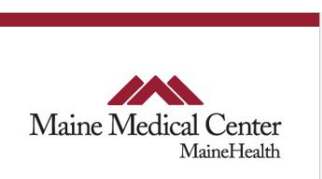




\section{Trends in Diagnoses of Neonatal Abstinence Syndrome at Newborn Hospitalization in Maine, 2009-2018}

\section{Acknowledgements}

We thank the Maine Health Data Organization for providing data on inpatient hospitalizations in Maine.

Authors

Emily C. Bauer MPH; Katherine Ahrens PhD; and Jenny L. Carwile MPH, ScD 


\title{
ORIGINAL RESEARCH
}

\section{Trends in Diagnoses of Neonatal Abstinence Syndrome at Newborn Hospitalization in Maine, 2009-2018}

\author{
Emily Bauer, MPH, RN, ${ }^{1}$ Jenny L Carwile MPH, $\mathrm{ScD},{ }^{2}$ Katherine Ahrens $\mathrm{PhD}^{1}$ \\ ${ }^{1}$ University of Southern Maine, Muskie School of Public Service, Portland, ME, ${ }^{2}$ Maine Medical Center Research Institute, \\ Scarborough, ME
}
Introduction: Incidence rates of neonatal abstinence syndrome (NAS) have increased in Maine, but whether this increase can be explained by use of different diagnosis codes over time is unknown. Our objective was to estimate trends in diagnoses of NAS at newborn hospitalization in Maine using different NAS case definitions.
Methods: $\quad$ We used International Classification of Diseases (ICD) diagnosis codes to identify newborns diagnosed with NAS in Maine between 2009 and 2018 using state-level hospital discharge data $(\mathrm{n}=123519)$. First, we considered only ICD-9 and ICD-10 codes used for confirmed NAS. Then we used an expanded ICD- 10 NAS case definition that included codes for neonates suspected to have NAS and affected by an unspecified "other maternal medication." We used joinpoint regression to model trends over time and identified changes in slope.

Results: $\quad$ Using the case definition for confirmed NAS, diagnoses of NAS increased from 2009 to 2013 (from 20
to 38 per 1000 births), and then decreased from 2013 to 2018 (from 38 to 28 per 1000 births). Using
our expanded ICD-10 NAS case definition, NAS increased linearly from 2009 to 2018 (from 24 to 50 per
1000 births).

Discussion: $\quad$ The trends in diagnoses of NAS were different when based on the expanded or confirmed NAS case definition.

Conclusions: Validation studies are needed to understand which infants are coded using the expanded versus confirmed NAS case definition. Caution should be used when interpreting rates of NAS in Maine using hospital discharge data.

Keywords: $\quad$ neonatal abstinence syndrome, International Classification of Diseases, ICD-9, ICD-10

$\mathrm{N}$ eonatal abstinence syndrome (NAS) is a condition of withdrawal symptoms in infants that is most commonly caused by exposure to opioids in utero. ${ }^{1}$ Rates of NAS increased more than 5-fold in the United States from 2004 to 2014 (from 1.5 to 8.0 per 1000 hospital births). ${ }^{2}$ Maine had the second highest annual increase in incidence of NAS between 1999 and 2013, with incidence rates increasing from 1.1 to 30.4 per 1,000 hospital births during 1999 to $2012 .{ }^{3}$ Current data are needed to estimate state-level trends in NAS in Maine after 2012. Also, while NAS surveillance and

Correspondence: Katherine Ahrens, PhD

University of Southern Maine, Muskie School of Public Service 34 Bedford Street, Portland, ME 04101

katherine.ahrens@maine.edu other research ${ }^{2,4,5}$ often identifies NAS cases from hospital billing data using diagnosis codes, little is known about how the use of different diagnosis codes for NAS has changed over time.

Recent coding changes for NAS may have introduced discrepancies in NAS classification over time. On October 1, 2015, the International Classification of Diseases changed the diagnosis codes used to identify NAS in-hospital discharge data from Clinical Modification ninth revision (ICD9) to the tenth revision (ICD-10). In 2018, certain NAS-related diagnosis codes were introduced or expanded to capture more detailed information on neonatal in utero exposure to maternal medication and drug use.$^{6,7}$ While ICD-9 and ICD-10 diagnosis 
codes for NAS were found to have high positive predictive value (> 90\%) in a study validating Tennessee Medicaid data against medical records, ${ }^{8}$ ICD-9/10 codes have not been validated more broadly and may vary in accuracy depending on the true prevalence of NAS and other factors. States lack standardized definitions of and surveillance practices for NAS, which could lead to different estimates for this condition across the United States. ${ }^{9}$

Our objective was to estimate trends in diagnoses of NAS at newborn hospitalization in Maine using state-level hospital discharge data. We examined trends using 3 NAS case definitions created based on ICD-9/10 diagnosis codes: 1) a primary definition, which was restricted to confirmed cases; 2) a secondary definition, which included neonates suspected to have NAS; and 3) an expanded ICD10 definition, which additionally included codes for unspecified maternal drug use during pregnancy. We also evaluated whether newborns defined as having NAS using the confirmed/suspect versus expanded ICD-10 case definitions differed according to medical and hospital characteristics.

\section{METHODS}

\section{Data source}

We examined newborn hospitalizations in Maine from 2009 to 2018 using individual-level inpatient discharge data provided by the Maine Health Data Organization, a state agency that maintains a comprehensive health information database of every inpatient hospital encounter in the state. ${ }^{10}$ The University of Southern Maine's Institutional Review Board approved the study protocol.

\section{NAS identification}

First, we identified newborn hospitalizations by restricting hospital discharge data to persons under 1 year of age with an ICD diagnosis code indicating a live birth ${ }^{11}$ or whose date of birth matched the date of hospital admission. We then compared the number of births we identified each year from the hospital discharge data to the total number of births in hospitals or clinical settings among Maine residents each year. ${ }^{12}$
Next, we scanned hospital reported diagnosis codes for any code that matched our NAS definitions. For ICD-10 (October 2015 through 2018), we used the 2019 Council of State and Territorial Epidemiologists (CSTE) Tier 2 case definition for confirmed and suspect NAS when using administrative data (see CSTE reference's Appendix 4). ${ }^{6}$ Our primary NAS case definition was the 'confirmed and probable' CTSE NAS case definition ICD-10 codes (P96.1, neonatal abstinence syndrome). As a secondary definition, we additionally included the CSTE 'suspect' NAS case definition ICD-10 codes (P04.14, newborn affected by maternal use of opiates; P04.17, newborn affected by maternal use of sedative-hypnotics; and P04.1A, newborn affected by maternal use of anxiolytics) (Table 1). For the third definition, we reviewed the NAS-related 'of interest' codes compiled by CSTE to identify codes for unspecified maternal medication or drug use that may have indicated in utero exposure to opioids. These codes could have been used to capture polysubstance use involving opioids or medication-assisted treatment with opioids, neither of which is specifically listed as an ICD-10 in utero exposure code. The third definition, which we called 'expanded ICD-10', therefore included our confirmed and suspect NAS case definition codes plus codes for newborns that indicated they were affected by unspecified maternal medication or drug use (Table 1).

The CSTE case definitions for NAS do not include ICD-9 codes; therefore, we selected ICD-9 codes for each NAS definition that were consistent with our 3 ICD-10 definitions. For the primary definition, we used ICD-9 (2009 through September 2015) code 779.5 , which was used in previous studies to identify confirmed cases. ${ }^{8,13}$ For both the suspect and expanded NAS case definitions, we also included codes 779.4 (drug reactions and intoxications specific to newborn) and 760.72 (narcotics affecting fetus or newborn via placenta or breast milk). These codes align with ICD-10 codes for suspect NAS and unspecified maternal medication/drug use. Code 760.72 was previously identified as an ICD-9 code equivalent for 2 of the ICD-10 codes used in our expanded ICD-10 definition (P04.40 and P04.49). ${ }^{14}$ 
Table 1. ICD-9/ICD-10 Diagnosis Codes Used for Primary and Secondary Definitions of Neonatal Abstinence Syndrome.

\section{NAS case definition \\ Primary case definition}

- Confirmed NAS

Secondary case definition

- Confirmed or suspect NAS*

ICD-10

ICD-9

ICD-10

- Drug withdrawal syndrome in newborn

P96.1 - NAS

779.5 - Drug withdrawal syndrome in newborn

P96.1 - NAS

779.4 - Drug reactions and intoxications specific to newborn

760.72 - Narcotics affecting fetus or newborn via placenta or breast milk

P04.14 - Newborn affected by maternal use of opiates

P04.17 - Newborn affected by maternal use of sedative-hypnotics

P04.1A - Newborn affected by maternal use of anxiolytics

Expanded ICD-10 definition

- Confirmed or suspect NAS*

ICD-9

779.5 - Drug withdrawal syndrome in newborn

779.4 - Drug reactions and intoxications specific to newborn

760.72 - Narcotics affecting fetus or newborn via placenta or breast milk

ICD-10 P96.1 - NAS

P04.14 - Newborn affected by maternal use of opiates

P04.17 - Newborn affected by maternal use of sedative-hypnotics

P04.1A - Newborn affected by maternal use of anxiolytics

- Unspecified newborn affected ICD-10

P04.1 - Newborn affected by other maternal medication

P04.18 - Newborn affected by other maternal medication $\neq$

P04.19 - Newborn affected by maternal use of unspecified medication $\neq$

P04.40 - Newborn affected by maternal use of unspecified drugs of addiction $\neq$

P04.49 - Newborn affected by maternal use of other drugs of addiction

Abbreviations: ICD, International Classification of Diseases; NAS, neonatal abstinence syndrome.

* ICD-10 codes for "Confirmed or Suspect Case", Appendix 4.6

+ Subset of ICD-10 codes for "Exposed but no clinical signs of withdrawal", Appendix $5 .^{6}$

$\ddagger$ ICD-10 codes introduced in October 2018; P04.18 and P04.19 are 2 of 9 expansion codes of the existing code P04.1 
Table 2. Characteristics of Newborns according to Categorization by ICD-10 Definition of Neonatal Abstinence Syndrome: Maine hospitals, October 2015-December $2018(n=39021)$.

\begin{tabular}{|c|c|c|c|c|}
\hline & $\begin{array}{c}\text { Confirmed or suspect } \\
\text { NAS, } \\
\text { No. }(\%)\end{array}$ & $\begin{array}{l}\text { Unspecified maternal } \\
\text { drug use codes and } \\
\text { no confirmed or } \\
\text { suspect NAS, } \\
\text { No. }(\%)\end{array}$ & $\begin{array}{c}\text { Comparison of } \\
\text { confirmed/ } \\
\text { suspect NAS } \\
\text { vs unspecified } \\
\text { maternal drug } \\
\text { codes only }\end{array}$ & $\begin{array}{l}\text { No confirmed NAS, } \\
\text { suspect NAS, or } \\
\text { unspecified maternal } \\
\text { drug use codes, } \\
\text { No. }(\%)\end{array}$ \\
\hline & $(n=1247)$ & $(n=999)$ & $P$ value & $(n=36775)$ \\
\hline \multicolumn{5}{|l|}{ Birth year } \\
\hline 2015 & $104(8.3)$ & $80(8.0)$ & .55 & $2778(7.6)$ \\
\hline 2016 & $423(33.9)$ & $342(34.2)$ & & $11521(31.3)$ \\
\hline 2017 & $382(30.6)$ & $329(32.9)$ & & $11201(30.5)$ \\
\hline 2018 & $338(27.1)$ & $248(24.8)$ & & $11275(30.7)$ \\
\hline Rurality of residence* & & & $<.001$ & \\
\hline Metro & $435(34.9)$ & $261(26.1)$ & & $13078(35.6)$ \\
\hline Large rural & $387(31.0)$ & $366(36.6)$ & & $13371(36.4)$ \\
\hline Small rural & $350(28.1)$ & $269(26.9)$ & & $7985(21.7)$ \\
\hline Isolated rural & $62(5.0)$ & $88(8.8)$ & & $1816(4.9)$ \\
\hline Other/out of state & $13(1.0)$ & $15(1.5)$ & & $522(1.4)$ \\
\hline \multicolumn{5}{|l|}{ Preterm birth } \\
\hline Yes & $166(13.3)$ & $201(20.1)$ & $<.001$ & $3054(8.3)$ \\
\hline No & $1081(86.7)$ & $798(79.9)$ & & $33721(91.7)$ \\
\hline \multicolumn{5}{|c|}{ Intrauterine growth restriction } \\
\hline Yes & $156(12.5)$ & $107(10.7)$ & .19 & $1399(3.8)$ \\
\hline No & $1091(87.5)$ & $892(89.3)$ & & 35376 (96.2) \\
\hline \multicolumn{5}{|l|}{ In-hospital infant death } \\
\hline Yes & Suppressed & 0 & NC & $102(0.3)$ \\
\hline No & $1246(99.9)$ & $999(100.0)$ & & $36673(99.7)$ \\
\hline
\end{tabular}


Table 2. (continued)

Any codes for "affected by"

Maternal tobacco use, P04.2

Maternal cannabis use, $P 04.81$

Maternal alcohol use, P04.3

Maternal antidepressant use, $P 04.15^{\dagger}$

Maternal amphetamine use, $P 04.16^{\dagger}$

All other maternal drug use ${ }^{\ddagger}$

Length of stay

Mean (SE)

Median (interquartile range)

Readmission within 1 year (rate per 1000 births) $)^{\S}$

Hospital

Northern Light Eastern Maine Medical Center

Maine Medical Center

MaineGeneral Medical Center

St. Mary's Regional Medical Center

Central Maine Medical Center

All other hospitals"

$193(15.5)$
Suppressed
Suppressed
0
0
$25(2.0)$
$10.2(0.26)$
$6.0(5.0,13.0)$

72 (57.8)

432 (34.6)

$281(22.5)$

$129(10.3)$

$66(5.3)$

$60(4.8)$

$279(22.4)$

$233(23.3)$
Suppressed
$10(1.0)$
0
0
$20(2.0)$

$<.001$

NC

$<.01$

$\mathrm{NC}$

$\mathrm{NC}$

.99

$<.001$

$5.4(0.22)$

$3.0(2.0,6.0)$

35 (35.1)

.01

$306(0.8)$

$21(0.1)$

$12(0)$

Suppressed

0

$60(0.2)$

$3.6(0.04)$

$2.0(2.0,3.0)$

$1248(33.9)$

$\begin{array}{cc}62(6.2) & <.001 \\ 293(29.3) & <.01 \\ 125(12.5) & .11 \\ 19(1.9) & <.001 \\ 80(8.0) & <.01 \\ 420(42.0) & <.001\end{array}$

5314 (14.5)

8980 (24.4)

$3104(8.4)$

$1827(5.0)$

1946 (5.3)

$15604(42.4)$

Abbreviations: NAS, neonatal abstinence syndrome; NC, not calculated due to small cell size; SE, standard error.

Suppressed cells indicate $\mathrm{n} \leq 10$ observations.

* Rural-urban designations were determined using a 4-category classification based on 2010 rural-urban community area codes, a census tract-based classification system: all metro $(1,1.1)$; large rural $(2,2.1,3,4,4.1,5,5.1,6)$; small rural $(7,7.1,7.2,8,8.1,8.2,9,10.1,10.2,10.3)$; and isolated rural $(10)$. Three observations were missing zip code information.

†These ICD-10 codes were introduced in October 2018 and are 2 of 9 expansion codes of the existing code P04.1.

‡ Separate ICD-10-CM codes exist for newborns affected by maternal use of chemotherapy, cytotoxic drugs, anticonvulsants, sedative-hypnotics, anxiolytics, cocaine, hallucinogens, nutritional chemical substances, environmental chemical substances, noxious substances, other maternal noxious substances, and unspecified noxious substances.

$\S$ Potential duplicate newborn hospitalizations $(n=8)$ were removed from the readmission rate denominator.

"Other hospitals include: Bridgton Hospital, Northern Light Mercy Hospital, Redington-Fairview General Hospital, Waldo County General Hospital, Rumford Hospital, Norther Light AR Gould Hospital, Southern Maine Health Care, York Hospital, Houlton Regional Hospital, Down East Community Hospital, Cary Medical Center, Stephens Memorial Hospital, Franklin Memorial Hospital, Mount Desert Island Hospital, Northern Light Inland Hospital, Mid Coast Hospital, Northern Light Maine Coast Hospital, Northern Maine Medical Center, Calais Regional Hospital, Pen Bay Medical Center, Mayo Regional Hospital, and LincolnHealth. 


\section{Newborn medical and hospital characteristics}

We compared several medical and hospital characteristics between newborns who were identified as having NAS using the confirmed/ suspect case definition and the expanded ICD-10 case definition. We restricted this comparison to October 2015 through December 2018 because, by design, it was only after the ICD-9/ICD-10 transition that the expanded definition identified a unique group of newborns. We used diagnosis codes to categorize infants by preterm birth (< 37 completed weeks' gestation) and intrauterine growth restriction using previously published code lists and online ICD-9/10 crosswalks. ${ }^{15-18}$ We also scanned diagnosis codes to identify newborns affected by maternal tobacco use, cannabis use, alcohol use, antidepressant use, amphetamine use, and all other maternal drug use codes (see CSTE Appendix 5). ${ }^{6}$ We then categorized infants by year of birth, hospital of birth, length of newborn hospitalization stay, and if they died during their newborn hospitalization (using discharge status codes). We used residential zip code to categorize infants by rurality of residence based on the 2014 rural tier assignments published by the New England Rural Health Roundtable. ${ }^{19}$ These assignments use the 2010 United States Department of Agriculture Rural-Urban Commuting Area codes, a census tract-based classification system that takes into account population density, urbanization, and daily commuting. ${ }^{20}$ Finally, we calculated the rate of readmission to the birth hospital within the first 12 months of life. Only readmissions to the birth hospital could be reliably identified in these data because different hospitals use different identifying numbers for each patient.

\section{Statistical analysis}

Linear trends in the rate of NAS at newborn hospitalization were estimated using Joinpoint Regression Program 4.8.01. We allowed for up to 2 joinpoints (ie, changes in slope) to be detected, and specified that the software calculate crude rates (and standard errors) per 1000 births and plot trends without log transformation. We analyzed differences in select medical and hospital characteristics of the newborns by NAS case definitions with chi-square and $t$-tests using SAS version 9.4 (SAS Institute, Cary, NC).

https://knowledgeconnection.mainehealth.org/jmmc/vol3/iss1/4 DOI: $10.46804 / 2641-2225.1071$

\section{RESULTS}

We identified 123519 infants from 2009 to 2018 Maine hospital data, which corresponded closely with the number of births in hospitals/clinical settings from 2009 to 2018 for Maine residents ( $\mathrm{n}$ $=124798) \cdot{ }^{12}$

Figure 1 shows the time trends from the joinpoint regression analyses for the 3 NAS case definitions. Using our primary NAS case definition (confirmed cases), diagnoses of NAS increased from 2009 to 2014 (observed: 20 to 38 per 1000 births; annual percentage increase: 4.0 per 1000 births [segment linear trend $P<.01])$. It then decreased from 2014 to 2018 (observed: 38 to 28 per 1000 births; annual percentage decrease: 2.5 per 1000 births $[P<$ .01]) (Figure 1A). Using our secondary NAS case definition (confirmed/suspect cases), diagnoses of NAS increased from 2009 to 2013 (observed: 24 to 43 per 1000 births; annual percentage increase: 5.3 per 1000 births $[P<.01])$, and then decreased from 2013 to 2018 (observed: 43 to 29 per 1000 births; annual percentage decrease: 3.2 per 1000 births $[P$ $<.01]$ ) (Figure 1B). In contrast, when we used our expanded ICD-10 NAS definition, diagnoses of NAS increased linearly from 2009 to 2018 (observed: 24 to 50 per 1000 births; annual percentage increase: 3.8 per 1000 births $[P<.01])$ (Figure $1 \mathrm{C})$.

Of the 39021 newborn hospitalizations with ICD10 codes (October 2015 to December 2018), 1240 (3.2\%) met the primary NAS definition, 1247 (3.2\%) met the secondary NAS definition, and 2246 (5.8\%) met the expanded ICD-10 NAS definition (Figure $2)$. We compared newborns who met the secondary definition (confirmed/suspect cases) ( $n=1247$ ) to those identified only by unspecified maternal drug use codes ( $n=999$; a subset of the expanded ICD10 definition group). The latter were more likely to be born preterm ( $13 \%$ vs $20 \% ; P<.001)$, more likely to have a diagnosis code for newborn affected by maternal tobacco use (16\% vs $23 \%$; $P<.001)$, had a shorter length of stay for newborn hospitalization (10.2 vs 5.4 mean days; $P<.001$ ), and had a lower rate of at least one hospital readmission within the first 12 months of life (58 vs 35 per 1000 births; $P<.01)$ (Table 2). They were also more likely to among the infants born at Maine Medical Center (23\% vs $30 \% ; P<.001$ ) or Central Maine Medical Center ( $5 \%$ vs $8 \% ; P<.001)$, and less likely to be among the infants born at Northern Light Eastern Maine Medical Center (35\% vs 6\%; $P<.001$ ) and St. Mary's Regional Medical Center (5\% vs $2 \%$; $P$ $<$.001) (Table 2). 
A. Primary definition (confirmed NAS)

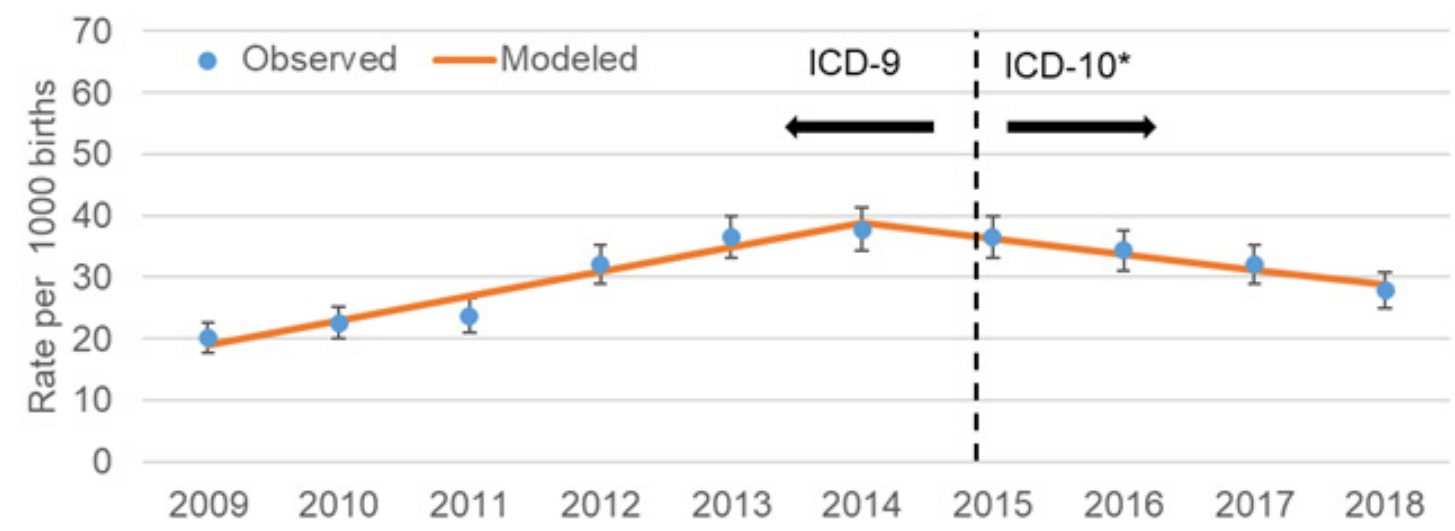

B. Secondary definition (confirmed or suspect NAS)

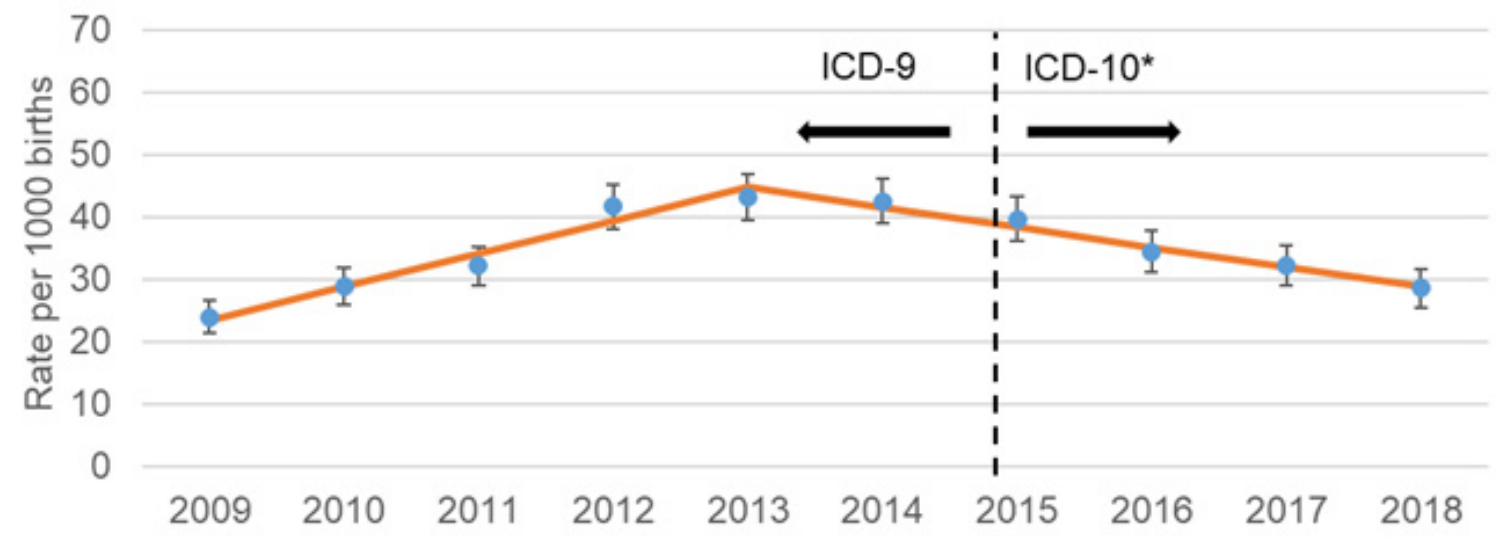

C. Expanded ICD-10 definition (includes unspecified maternal drug use codes)

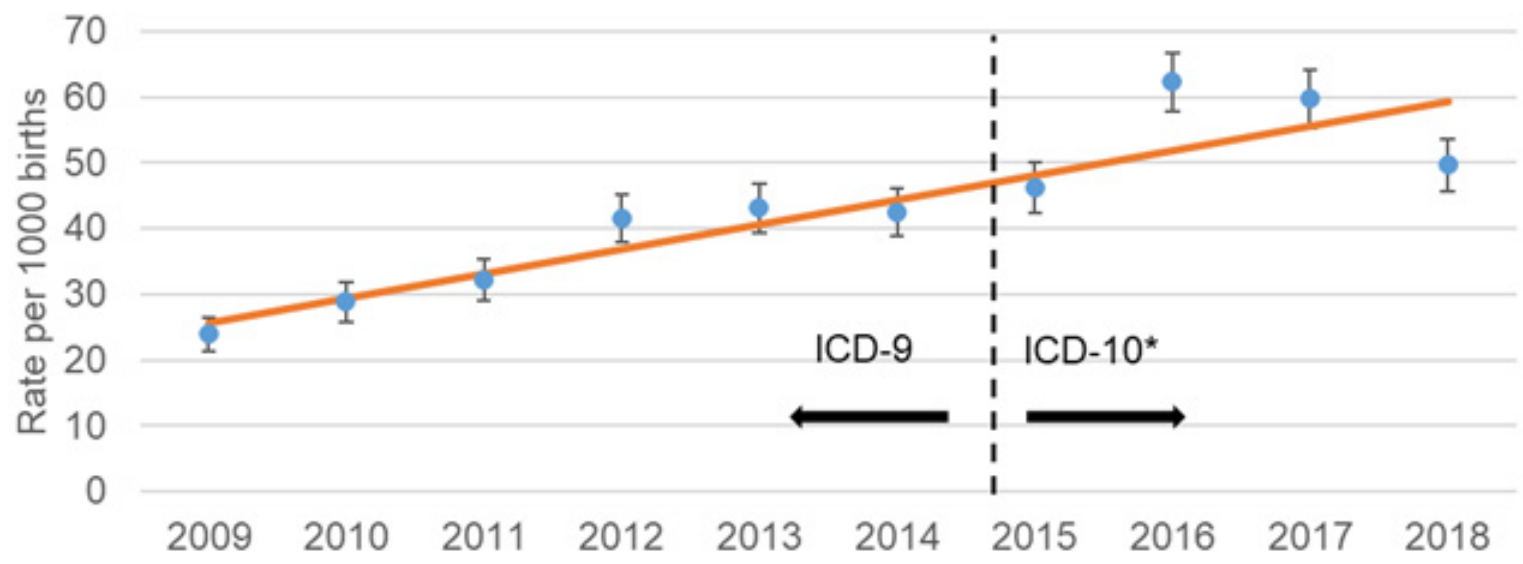

Figure 1. Observed and modeled rate of neonatal abstinence syndrome diagnosis among Maine hospital births using 3 ICD diagnosis code definitions, 2009-2018 $(n=123519)$. Vertical dashed line indicates the data transition from ICD-9 to ICD-10 on October 1, 2015. 
Journal of Maine Medical Center, Vol. 3 [2021], Iss. 1, Art. 4

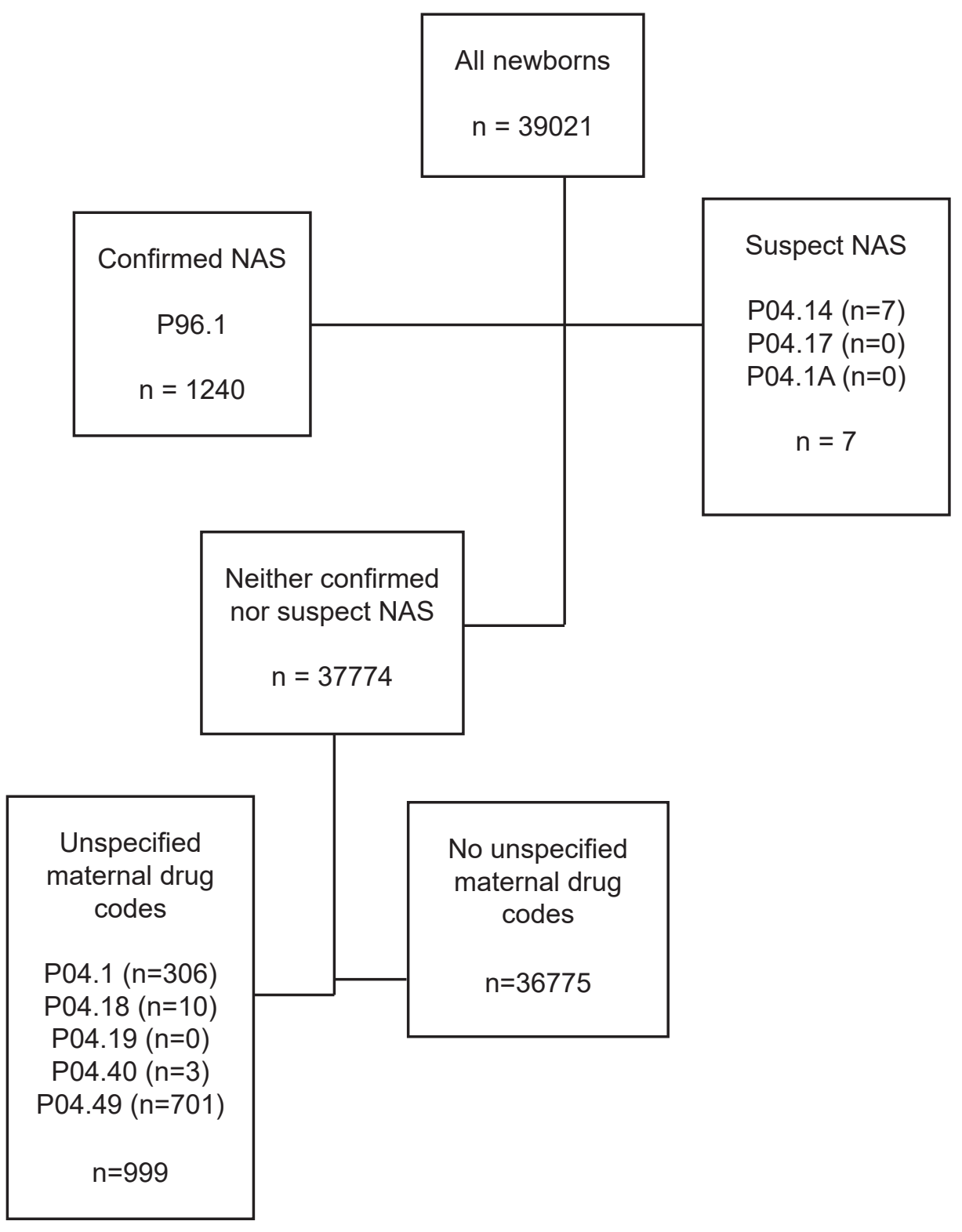

Figure 2. Flow chart showing categorization of newborns by ICD-10 neonatal abstinence syndrome definition: Maine hospitals, October 2015 to December 2018. NAS, neonatal abstinence syndrome. 


\section{DISCUSSION}

We found that trends in NAS were different based on whether the ICD-10 diagnosis codes used to define NAS included codes used for newborns affected by unspecified maternal drug/medication use. When we excluded those codes, the incidence of NAS increased from 2009 to 2013/2014 and then decreased from 2013/2014 to 2018. When we included those codes, NAS showed a linear increase from 2009 to 2018, more than doubling from a predicted incidence of 26 to 59 per 1000 births (observed: 24 to 50 per 1000 births), with an annual percentage increase of 3.8 per 1000 births. Trends for confirmed and confirmed/suspect NAS were similar. Several characteristics of infants with only unspecified maternal drug use codes were different from those of infants with confirmed/ suspect NAS. These differences include having a higher risk of preterm birth, being more likely to have exposure to maternal tobacco use, and having a shorter newborn hospitalization and readmission rate. They also include being more likely to be among the infants born at Maine Medical Center and Central Maine Medical Center, and less likely to be among the infants born at Northern Light Eastern Maine Medical Center and St. Mary's Regional Medical Center.

Our findings regarding the confirmed/suspect NAS case definition are generally consistent with previous findings, supporting the absence of artifactual changes in NAS diagnosis around the time of the ICD-9/ICD-10 transition (October 2015). A recent publication examined national trends in the number of NAS births between 2008 and 2019, using ICD-10 codes for confirmed NAS plus one of the suspect ICD-10 codes (P04.14; newborn affected by maternal use of opiates), ${ }^{21}$ and found the number of NAS cases in the United States increased until 2017, after which it decreased. In Maine, we found a decrease in confirmed NAS cases beginning in 2014. We expected that an artifactual change in the rate of NAS due to the ICD9/ICD-10 transition would change the trend in 2015 or 2016, but we did not observe this effect. A true decline in NAS cases in Maine since 2014 is also supported by studies in Tennessee, ${ }^{8}$ Illinois, New Mexico, Vermont ${ }^{22}$ and Florida ${ }^{13}$. These studies compared NAS cases identified from hospital discharge data to medical record data (as the gold standard), and they found high positive predictive values for the confirmed NAS case definition codes across the ICD-9/ICD-10 transition. Although the more granular nature of the tenth revision codes may allow for the correct assignment of unspecified maternal drug/medication use codes that would have been erroneously assigned as confirmed NAS during ICD-9 (ie, false discovery rate), this explanation is not supported by the high positive predictive value observed across the ICD-9/ICD-10 transition. ${ }^{8,13,22}$

Data from Illinois, New Mexico, Vermont ${ }^{22}$ and Florida $^{13}$ also suggest that cases identified using diagnosis codes for confirmed NAS were more likely to be true NAS cases than those identified using other diagnosis codes, including 760.72 (noxious influences affecting fetus or newborn placenta or breast milk, narcotics). These findings suggest that some of the cases we identified using diagnosis codes for suspect or unspecified NAS may not have actually been NAS cases. The other ICD-9/ICD-10 diagnosis codes we used to define suspect NAS cases and the ICD-10 codes we used to identify expanded ICD-10 NAS cases have yet to be validated.

Our findings for confirmed/suspect NAS cases generally align with those from a recent trend analysis of the prevalence of opioid use disorder among women delivering in Maine hospitals from 2009 to 2018 (using the same data source). ${ }^{23}$ Although a linear increase of 1.6 cases per 1000 deliveries per year was detected among women (from 22.7 to 34.9 per 1000 deliveries $[P<.01]$ ), non-linear trends were not investigated. Thus, it is possible that there was a change in slope around 2013 that mirrored what we observed in this study. In utero exposure to opioids in pregnancy, whether for treatment or due to illicit use, is associated with a $60 \%$ to $80 \%$ risk of NAS, and long-term childhood health outcomes are largely unknown. Monitoring the prevalence of maternal opioid use disorder is therefore one way to assess trends in NAS. ${ }^{24,25}$

Thus, one explanation for our findings is that unspecified drug codes (eg, P04.40 and P04.49) may have been used for infants thought to be exposed to opioids in utero, but who did not display signs or symptoms of drug withdrawal and therefore were not true NAS cases. However, coding exposed but asymptomatic newborns with a maternal medication/drug use code would be inconsistent with billing practice recommendations, which state that use of any 'newborn affected by' diagnosis code be reserved for newborns 
displaying signs or symptoms of drug withdrawal following in utero exposure. ${ }^{7}$ The long descriptions of the maternal medication and drug use diagnosis codes that were introduced in ICD-10 initially contained the qualifier 'suspected to be' before 'affected'. This qualifier may have encouraged the practice of coding asymptomatic infants thought to be exposed to opioids with these unspecified maternal drug use codes. ${ }^{14}$ This scenario could explain the sudden increase in NAS diagnoses (which included P04.40 and P04.49) observed in Missouri after the ICD-9/ICD-10 transition. ${ }^{14}$ Coding exposed but asymptomatic infants with the unspecified drug codes is further supported by our observation that infants identified as having NAS based on unspecified ICD-10 codes alone tended to be hospitalized for a shorter period and were less likely to be readmitted within 1 year compared with infants with confirmed NAS. ${ }^{2,20}$

An alternative explanation for our findings is that symptomatic infants suspected of exposure to opioids in utero were coded with the unspecified drug codes instead of the confirmed/suspect NAS codes starting in the ICD-10 period. One reason could be because there are no specific codes for symptomatic infants exposed in utero to maternal medication-assisted treatment for opioid use disorder. Thus, in an attempt to differentiate these infants from those born to mothers with illicit opioid use, these cases may have been recorded in the billing claims data using one of the unspecified maternal drug use codes rather than the code for confirmed NAS (P96.1). ${ }^{7}$ This practice could have increased over time because of increases in the prevalence of medication-assisted treatment in pregnant women ${ }^{26}$ and in the general population. ${ }^{27}$ Similarly, it is possible that unspecified maternal drug use codes were used to distinguish infants with NAS treated using the Eat Sleep Console method, which was implemented by most Maine hospitals between 2018 and 2019,28 from those receiving standard pharmacological treatment. Infants born to mothers using medication-assisted treatment and those treated using Eat Sleep Console receive less pharmacological treatment and have shorter hospital stays on average than other infants with NAS. ${ }^{28}$ These data are consistent with our observation of shorter hospital stays in infants assigned unspecified maternal drug use codes. Finally, the rise in use of unspecified maternal drug use codes could be due to a recent increase (since 2015) in polysubstance use, drug use of unknown type, or use of another drug or medication among women delivering in Maine. Polysubstance use is common among opioid users, and co-use of methamphetamine increased between 2011 and 2018. ${ }^{29}$ Further, polysubstance use can influence withdrawal timing and severity in infants. ${ }^{30}$ Although medical cannabis was legalized for adult recreational use in Maine in $2017,{ }^{31}$ we considered cannabis use unlikely to account for the increase in use of unspecified drug codes because there is an ICD-10 code specifically for newborns affected by maternal cannabis use (P04.81).

Hospital discharge data are convenient for monitoring trends in NAS because they are multiyear population-based administrative data. They represent all hospitalizations at the state or national-level, are free or of low cost to use, and represent nearly all infants born in the state each year. ${ }^{32}$ However, before incorporating unspecified maternal drug use codes into an expanded ICD10 NAS case definition, we recommend additional validation of these cases. This validation could include electronic and paper-based chart reviews, as well as interviews with medical records staff.

Our study has several strengths and limitations. Strengths include our examination of trends for multiple case definitions for NAS following recent CSTE recommendations for case definitions. ${ }^{6}$ We provide 6 years of updated estimates for NAS incidence in Maine, beyond the most recently published estimates, ${ }^{3}$ which is critical for making up-to-date assessments of the public health burden of NAS in Maine. However, our data represent only one state, and different patterns in NAS coding may occur in other states due to factors including education of medical staff regarding NAS coding, trends in polysubstance use, and pharmacological treatment for NAS.

\section{CONCLUSIONS}

Due to differences in trends and characteristics observed between infants identified using confirmed and more expanded NAS definitions, caution should be used when interpreting surveillance or research findings for NAS in Maine and possibly other states based on hospital discharge data alone. This caution is particularly important for findings that incorporate expanded ICD-10 case definitions for NAS. Validation of billing codes against hospitallevel electronic medical records is needed to accurately assess the incidence rate of NAS among 
newborns, while also taking into account changes in coding, updates to clinical practices related to treatment of mothers and infants, and trends in substance use.

Acknowledgments: We thank the Maine Health Data Organization for providing data on inpatient hospitalizations in Maine.

\section{Conflict of interest: None}

Disclosures: The views expressed in this article are those of the authors and not an official position of any institution or funder.

Funding: E.B. and K.A.A. performed this work with funding from the Maine Economic Improvement Fund (MEIF). MEIF was not involved in study design; collection, analysis, and interpretation of data; writing of the report; or publication decisions.

\section{REFERENCES}

1. McQueen K, Murphy-Oikonen J. Neonatal abstinence syndrome. $N$ Engl J Med. 2016;375(25):2468-2479. doi:10.1056/ NEJMra1600879

2. Winkelman TNA, Villapiano N, Kozhimannil KB, Davis MM, Patrick SW. Incidence and costs of neonatal abstinence syndrome among infants with Medicaid: 2004. Pediatrics. 2018;141(4):e20173520. doi:10.1542/peds.2017-3520

3. Ko JY, Patrick SW, Tong VT, Patel R, Lind JN, Barfield WD. Incidence of neonatal abstinence syndrome - 28 states, 19992013. MMWR Morb Mortal Wkly Rep. 2016;65(31):799-802. doi:10.15585/mmwr.mm6531a2

4. Corr TE, Hollenbeak CS. The economic burden of neonatal abstinence syndrome in the United States. Addiction. 2017;112(9):1590-1599. doi:10.1111/add.13842

5. Patrick SW, Davis MM, Lehmann CU, Cooper WO. Increasing incidence and geographic distribution of neonatal abstinence syndrome: United States 2009 to 2012. J Perinatol. 2015;35(8):650-655. doi:10.1038/jp.2015.36

6. Council of State and Territorial Epidemiologists. CSTE position statement. Neonatal Abstinence Syndrome Standardized Case Definition. Council of State and Territorial Epidemiologists; 2019.

7. Ernst KD, Makkar A. The opioid-exposed neonate: a review of the Oklahoma experience. J Okla State Med Assoc. 2018;111(8):768774. Accessed September 17, 2020. https://www.ncbi.nlm.nih. gov/pmc/articles/PMC6660011/

8. Maalouf FI, Cooper WO, Stratton SM, et al. Positive predictive value of administrative data for neonatal abstinence ayndrome. Pediatrics. 2019;143(1):e20174183. doi:10.1542/peds.20174183

9. Chiang KV, Okoroh EM, Kasehagen LJ, Garcia-Saavedra LF, Ko JY. Standardization of state definitions for neonatal abstinence syndrome surveillance and the opioid crisis. Am J Public Health. 2019;109(9):1193-1197. doi:10.2105/AJPH.2019.305170

10. Maine Health Data Organization. Maine Rule Chapters for Independent Agencies, Title 22, Chapter 1683. Maine Health Data Organization; 2020.
11. Agency for Healthcare Research and Quality. Prevention Quality Indicator 09 (PQI 09) Low Birth Weight Rate. AHRQ QITM ICD-10-CM/PCS Specification Enhanced Version 5.0. U.S. Department of Health and Human Services; 2016.

12. Maternal and Birth Characteristics 2009-2018. Maine Center for Disease Control and Prevention. https://www.maine.gov/dhhs/ mecdc/public-health-systems/data-research/vital-records/births. shtml. Accessed September 17, 2020¥.

13. Phillips-Bell GS, Holicky A, Lind JN, et al. Assessing the burden of neonatal abstinence syndrome: validation of ICD-9CM Data, Florida, 2010-2011. J Public Health Manag Pract. 2020;26(1):E1-E8. doi:10.1097/PHH.0000000000000897

14. W Coffey, E Mobley, A Hunter. Tracking neonatal abstinence syndrome in Missouri: trends and the ICD-CM transition. Online Journal of Public Health Informatics. 2018;10(1). doi:10.5210/ ojphi.v10i1.8981

15. Kim HJ, Jo M-W, Bae S-H, Yoon S-J, Lee JY. Measuring the burden of disease due to preterm birth complications in Korea using disability-adjusted life years (DALY). Int J Environ Res Public Health. 2019;16(3):519. doi:10.3390/ijerph16030519.

16. Gilbert WM, Danielsen B. Pregnancy outcomes associated with intrauterine growth restriction. Am J Obstet Gynecol. 2003;188(6):1596-1599; discussion 1599-1601. doi:10.1067/ mob.2003.384

17. Nobles CJ, Grantz KL, Liu D, et al. Ambient air pollution and fetal growth restriction: physician diagnosis of fetal growth restriction versus population-based small-for-gestational age. Sci Total Environ. 2019;650(Pt 2):2641-2647. doi:10.1016/j. scitotenv.2018.09.362

18. The Web's Free ICD-9-CM Medical Coding Reference. ICD9Data.com. Accessed September 17, 2020. https://d19j0qt0x55bap.cloudfront.net/production/ onboardings/5de6c532499ed552c590886b/documents/file/3. The_Web_s_Free_ICD-9-CM_ICD-10-CM_Medical_ Coding_Reference.pdf

19. Rural Data for Action: A comparative analysis of health data for the New England region. New England Rural Health Roundtable; 2014.

20. Witt CE, Rudd KE, Bhatraju P, Rivara FP, Hawes SE, Weiss NS. Neonatal abstinence syndrome and early childhood morbidity and mortality in Washington state: a retrospective cohort study. $J$ Perinatol. 2017;37(10):1124-1129. doi:10.1038/jp.2017.106

21. Healthcare Cost and Utilization Project. Neonatal Abstinence Syndrome Births: Trends in the United States, 2008-2019. Agency for Healthcare Research and Quality; 2020.

22. Lind JN, Ailes EC, Alter CC, et al. Leveraging existing birth defects surveillance infrastructure to build neonatal abstinence syndrome surveillance systems - Illinois, New Mexico, and Vermont, 2015-2016. MMWR Morb Mortal Wkly Rep. 2019;68(7):177-180. doi:10.15585/mmwr.mm6807a3

23. Gabrielson SMB, Carwile JL, O'Connor AB, Ahrens KA. Maternal opioid use disorder at delivery hospitalization in a rural state: Maine, 2009-2018. Public Health. 2020;181:171-179. doi: 10.1016/j.puhe.2019.12.014

24. Honein MA, Boyle C, Redfield RR. Public health surveillance of prenatal opioid exposure in mothers and infants. Pediatrics. 2019;143(3):e20183801. doi:10.1542/peds.2018-3801

25. Stover MW, Davis JM. Opioids in pregnancy and neonatal abstinence syndrome. Semin Perinatol. 2015;39(7):561-565. doi:10.1053/j.semperi.2015.08.013

26. Krans EE, Kim JY, James AE, 3rd, Kelley D, Jarlenski MP. Medication-assisted treatment use among pregnant women with opioid use disorder. Obstet Gynecol. 2019;133(5):943-951. doi:10.1097/AOG.0000000000003231. 
27. Stein BD, Dick AW, Sorbero M, et al. A population-based examination of trends and disparities in medication treatment for opioid use disorders among Medicaid enrollees. Subst Abus. 2018;39(4):419-425. doi:10.1080/08897077.2018.1449166

28. Grossman MR, Lipshaw MJ, Osborn RR, Berkwitt AK. A novel approach to assessing infants with neonatal abstinence syndrome. Hosp Pediatr. 2018;8(1):1-6. doi:10.1542/hpeds.2017-0128

29. Cicero TJ, Ellis MS, Kasper ZA. Polysubstance use: a broader understanding of substance use during the opioid crisis. Am J Public Health. 2020;110(2):244-250. doi:10.2105/ AJPH.2019.305412

30. Sanlorenzo LA, Cooper WO, Dudley JA, Stratton S, Maalouf FI, Patrick SW. Increased severity of neonatal abstinence syndrome associated with concomitant antenatal opioid and benzodiazepine exposure. Hosp Pediatr. 2019;9(8):569-575. doi:10.1542/ hpeds.2018-0227

31. MAINE STATE LEGISLATURE. The following document is provided by the LAW AND LEGISLATIVE DIGITAL LIBRARY at the Maine State Law and Legislative Reference Library. http:// 1ldc.mainelegislature.org/Open/Laws/2017/2017_PL_c409.pdf Accessed September 17, 2020

32. MacDorman MF, Declercq E. Trends and state variations in out-of-hospital births in the United States, 2004-2017. Birth. 2019;46(2):279-288. doi: 10.1111/birt.12411 